\begin{tabular}{|c|c|}
\hline Title & $\begin{array}{l}\text { DeNovo Synthesis of Gold-Nanoparticle Embedded, Nitrogen-Doped Nanoporous Carbon Nanoparticles (A u@ NC) } \\
\text { with Enhanced Reduction A bility }\end{array}$ \\
\hline Author(s) & $\begin{array}{l}\text { Liao, Yu-Te; Chen, Jeffrey E.; Isida, Y ohei; Y onezawa, Tetsu; Chang, Wei-Chen; A Ishehri, Saad M.; Y amauchi, } \\
\text { Y usuke; Wu, Kevin C.-W. }\end{array}$ \\
\hline Citation & $\begin{array}{l}\text { ChemCatChem, 8(3), 502-509 } \\
\text { https://doi.org/10.1002/cctc.201501020 }\end{array}$ \\
\hline Issue Date & $2016-02-06$ \\
\hline Doc URL & http:/hdl. handle.net/2115/64467 \\
\hline Rights & $\begin{array}{l}\text { This is the accepted version of the following article: DeNovo Synthesis of Gold-Nanoparticle Embedded, Nitrogen- } \\
\text { Doped Nanoporous Carbon Nanoparticles (A u@ NC) with Enhanced Reduction A bility. ChemCatChem, 8(3):502- } \\
509 ., \text { which has been published in final form at http://dx.doi. org } 10.1002 / \text { ctc. } 201501020 \text {. }\end{array}$ \\
\hline Type & article (author version) \\
\hline File Information & Revised MS-cctc.201501020.pdf \\
\hline
\end{tabular}

Instructions for use 


\title{
De novo Synthesis of Au Nanoparticles-Embedded, Nitrogen-Doped Nanoporous Carbon Nanoparticles (Au@NC) with Enhanced Reduction Ability
}

\author{
Yu-Te Liao, ${ }^{[a]}$ Jeffrey E. Chen, ${ }^{[a]}$ Yohei Isida, ${ }^{[b]}$ Tetsu Yonezawa, ${ }^{*[b]}$ Wei-Chen Chang, ${ }^{[c]}$ Saad M. \\ Alshehri, ${ }^{[\mathrm{d}]}$ Yusuke Yamauchi, ${ }^{[\mathrm{e}]}$ and Kevin C. $-\mathrm{W} . \mathrm{Wu}^{\star{ }^{[a]}}$
}

A de novo synthesis of gold nanoparticles embedded, nitrogen doped nanoporous carbon nanoparticles (Au@NC) was synthesized in this work. The chloroauroic acid was encapsulated inside zeolitic imidazolate framework-8 (ZIF-8) nanoparticles during the synthesis and later reduced into gold nanoparticles. The as-synthesized gold nanoparticles embedded ZIF-8 (Au@ZIF-8) was then carbonized into Au@NC to enhance the stability of the nanoporous support. The results showed that Au@NC exhibited a porous structure containing 3wt\% of gold. 2-Methylimidazole provided abundant nitrogen (19wt\%) on the carbon matrix, resulting in hydrophilic and positive charge surface that is useful for the reduction of 4-nitrophenol. The results of the catalytic reaction indicated that the synthesized Au@NC could act as an effective catalyst with turnover frequency (TOF) of $1185 \mathrm{~g} \mathrm{~g}^{-1} \mathrm{~s}^{-1}$, which is higher than that of conventional naked Au nanoparticles (TOF of $339 \mathrm{~g}^{-1} \mathrm{~s}^{-1}$ ) and Au nanoparticles on activated carbon (TOF of $89 \mathrm{~g} \mathrm{~s}^{-1} \mathrm{~s}^{-1}$ ). We propose that the enhanced performance of the Au@NC resulted from the homogeneous distribution of Au nanoparticles along with the hydrophilic and positive charge surface of nitrogen-doped carbon surface.

Catalysis has been involved at the core of nearly all chemical protocols from scientific research to industrial applications. In general, catalysis could be divided into homogeneous and heterogeneous catalysis. Both of homogenous and heterogeneous catalysts have their own advantages and drawbacks. ${ }^{[1]}$ Some homogeneous catalysts have been tried to converted into hetrogeneous catalysts for easy recycling and other advantages. ${ }^{[2]}$

In recent years, not only organic functional groups but also organometallic compounds and metallic nanoparticles have been decorated on different supports to change homogeneous catalyst into heterogeneous catalyst. ${ }^{[3]}$ Mesoporous silica and zeolite are common supports for metal nanoparticles and organic functional groups since silica displays excellent

[a] Y.-T. Liao, J.-E. Chen, Prof. Dr. K. C.-W. Wu Department of Chemical Engineering

National Taiwan University

No. 1, Sec. 4, Roosevelt Road, Taipei 10617, Taiwan

Fax: (+886)2-2362-304

E-mail: kevinwu@ntu.edu.tw

[b] Dr. Y. Isida, Prof. Dr. T. Yonezawa

Department of Materials Science, Graduate School of Engineering, Hokkaido University,

Sapporo, 060-8628, Japan

E-mail: tetsu@eng.hokudai.ac.jp

[c] Dr. W.-C. Chang

Institute of Nuclear Energy Research,

Atomic Energy Council,

Taoyuan 32546, Taiwan

[d] Prof. Dr. S. -M. Alshehri

Department of Chemistry, College of Science,

King Saud University,

Riyadh 11451, Saudi Arabia

[e] Prof. Dr. Y. Yamauchi

World Premier International (WPI) Research Center for Materials, Nanoarchitectonics (MANA), National Institute for Materials Science (NIMS), 1-1 Namiki, Tsukuba, Ibaraki 305-0044, Japan

Supporting information for this article is available on the WWW under http://dx.doi.org/10.1002/cctc.20xxxxxxx. chemical and thermal stability, extreme accessibility, high surface area, and easy functionalization. ${ }^{[4]}$ As an example of such a recent development, Castro and co-workers functionalized ionic liquids on MCM-41 type mesoporous silica, which exhibited enhanced dodecene conversion efficacy as compared to that of the corresponding pure ionic liquids (i.e., $95 \%$ vs. 5\%). ${ }^{[5]}$ In addition to silica-based supports, metal oxides such as titania, magnesium oxide, and cerium oxide have also been utilized as effective supports for loading metal nanoparticles. ${ }^{[6]}$ Metal oxides are utilized due to the capability for oxygen vacancies in the oxides to reduce the activation energy of catalytic reactions. ${ }^{[7]}$ Other than silica and metal oxides, catalytic supports of carbon-based materials including activated carbon, mesoporous carbon, and graphite have also been widely used owing to their high stability towards acidic or basic environments. ${ }^{[8]}$ Moreover, another advantage of carbon-based supports is the possible interactions between the catalytically active species and the carbon surface. ${ }^{[9]}$ Despite these pioneering studies, the development of new nanoporous materials is still highly demanded for various applications.

Metal organic frameworks (MOFs) are a series of porous structures that are usually constructed by organic linkers and metal joints. ${ }^{[10]}$ The combination of various kinds of organic linkers and inorganic joints from different sources has led to more than thousands of MOFs with different physical and chemical properties. ${ }^{[11]}$ One of the advantages of MOFs over conventional zeolites and mesoporous materials is the extremely high specific surface area, which make MOFs attractive for various emerging applications, such as gas storage and catalysis. ${ }^{[12-14]}$ As for catalytic applications, the metal joints not only link the organic compounds but also serve as active sites for catalytic reactions. Direct carbonization of the synthesized MOF materials has been recently proposed. ${ }^{[15-18]}$ Through careful pyrolysis processes and different treatments, metal joints can either be removed from or remain in the framework while organic linkers convert to carbon; thus creating nanoporous carbon with loaded metal or metal oxide nanoparticles. $^{[19-20]}$ 

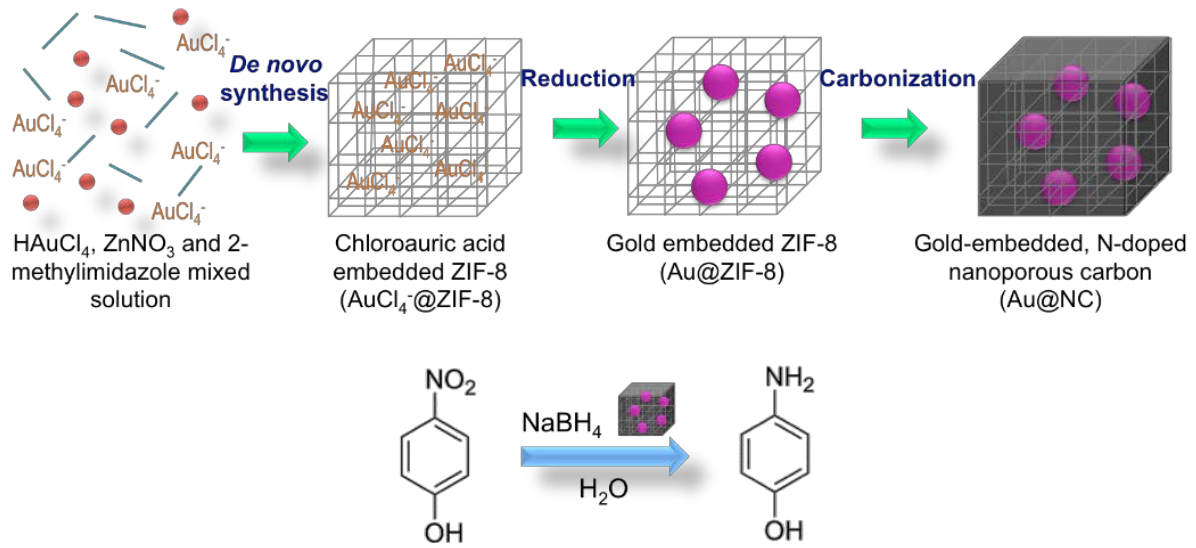

Scheme 1. De novo synthesis of Au nanoparticles-embedded, nitrogen-doped nanoporous carbon particles for reduction of 4-nitrophenol.

If the organic linkers contain nitrogen, there is even a possibility to produce nitrogen-loaded nanoporous carbon after carbonization of MOFs. ${ }^{[21]}$

There are many methods to load other metal species into MOFs to generate multi-functionalized catalysts, and in general they can be classified into the following three methods: chemical vapor infiltration, ${ }^{[22-23]}$ solution impregnation, ${ }^{[24-25]}$ and encapsulation of performed nanoparticles. ${ }^{[26-28]}$ The chemical vapor infiltration decomposes organometallic compounds into metal vapor that can be deposited in the structure of MOF supports. ${ }^{[23]}$ Although this method avoids the deficiencies of solution impregnation, high instrument cost and complicated operation processes make them less practical. Solution impregnation has been extensively applied for introducing guest species into porous supports owing to its easy procedure. ${ }^{[29]}$ The metal precursor penetrates into the porous structure of the MOF support by a concentration gradient to later be reduced into metal nanoparticles after the addition of a reducing agent, such as $\mathrm{NaBH}_{4}$ or hydrazine. ${ }^{[30-31]}$ However, it is difficult for the metal precursor to penetrate into the MOFs because the pore openings in the external surface of MOFs are smaller than those of mesoporous materials. For this reason, the metal precurser stays on the external surface of MOFs to result in inhomogeneous loading. Even if the metal precursor is capable of penetrating into the internal surface of MOFs, the reduction of metal ions (in other words, the formation of metal nanoparticles) could sometimes cause the collapses of the MOFs structure to result in broad particle size distribution. ${ }^{[32]}$ Encapsulation of performed metal nanoparticles to porous supports is the best method to control the location and the number of metal nanoparticles in MOFs. ${ }^{[33]}$ Nevertheless, it is always necessary to use surface-protecting agents (or so-called stabilizers) to reduce the particle size and to prevent each nanoparticle from aggregation. The presence of such stabilizers would inhibit the active sites of metal nanoparticles, resulting in low efficiency of metal-loaded MOF catalysts. ${ }^{[34]}$

Different from the methods mentioned above, we here report the de novo synthesis of metal nanoparticles-embedded zeolitic imidazolate framework-8 (ZIF-8) with gold ions introduced inside ZIF-8 upon the formation of ZIF-8 in aqueous solutions, as shown in Scheme 1 . The gold ions were successfully converted into gold nanoparticles in the voids of ZIF-8, resulting in gold nanoparticles with consistent particle size and homogeneous distribution inside ZIF-8. The gold nanoparticles embedded ZIF-8 (denoted as Au@ZIF-8) were then carbonized into nitrogen-containing nanoporous carbon (denoted as Au@NC), because ZIF-8 exhibits organic 2-methylimidazole groups that contain nitrogen. The synthesized Au@NC was used as a heterogeneous catalyst for the reduction of 4-nitrophenol. The results indicate that our Au@NC exhibited higher turnover frequency (TOF) than that of naked Au nanoparticles and gold-loaded activated carbon. We propose that the enhanced performance of the Au@NC resulted from the homogeneously suspended Au nanoparticles as well as the positive charge and hydrophilic surface of nitrogen-doped nanoporous carbon.

We first aim to study the effect of different amounts of gold ions on the synthesis of ZIF-8. SEM images of $\mathrm{AuCl}_{4}$-embedded ZIF-8 synthesized with different ratios of gold to zinc (from $0 \sim 0.2: 1$ ) are shown in Figure 1. It has been known that the formation of zeolitic imidazolate frameworks (ZIF) is based on the combination of the organic linker (i.e., 2-methylimidazole) and the metal joint (i.e., zinc nitrate hexahydrate). The organic components would lose a proton in the solution and then coordinate with the metal ions as a substitute for the proton to form nuclei. Therefore, any presence of guest ions would affect the formation of ZIFs crystal structure. In our case, $\mathrm{AuCl}_{4}{ }^{-}$ions were present in the solution where the ZIF-8 nanoparticles were synthesized. For comparison, pure ZIF-8 (i.e., without any addition of $\mathrm{AuCl}_{4}{ }^{-}$) was also synthesized, and its particle size was about $70 \mathrm{~nm}$ with sharp hexagonal facets (Figure 1a). When a small amount of $\mathrm{AuCl}_{4}^{-}$was added (the molar ratio of $\mathrm{Au}$ ions to $\mathrm{Zn}$ ions was 0.01 ), the obtained precipitate (denoted as $\mathrm{AuCl}_{4}{ }^{-} @ \mathrm{ZIF}-8$ ) displayed a particle size range similar to that of ZIF-8, but without the morphology of hexagonal facets (Figure 1b). When the molar ratio was further increased to 0.1 , the obtained sample exhibited irregular shape with particle aggregation, as shown in Figure 1c. This phenomenon was more apparent when the ratio was increased to 0.2 , where the average particle size increased to around 180 nm due to $\mathrm{AuCl}_{4}^{-} @ \mathrm{ZIF}-8$ particle aggregation, as shown in Figure 1d.

Since chloroauric acid solution is acidic, the $\mathrm{pH}$ value of the system would decrease with increasing ratios of chloroauric acid. We propose that such a $\mathrm{pH}$ decrease, in addition to the presence of $\mathrm{Au}$ ions, would affect the morphology of the final product. The key step for the nucleation of ZIF materials is the deprotonation of the organic component, and a more acidic 
environment would be less preferable for deprotonation. Guo et al. ${ }^{[35]}$ have found that in lower $\mathrm{pH}$ values, slow deprotonation would cause slow nucleation and crystal growth, resulting in larger particle size. The results obtained here also indicate that although the chloroauric acid would not be directly involved in the formation of ZIF-8 particles, the decreasing $\mathrm{pH}$ value caused by the addition of chloroauric acid affected the particle size and morphology of the final ZIF-8 particles. In contrast, the amount of $\mathrm{Au}$ is an important factor for using the synthesized Au-doped materials as an efficient catalyst. Therefore, to balance between particle size/morphology and catalytic performance, we chose the $\mathrm{AuCl}_{4}{ }^{-} @ \mathrm{ZIF}-8$ particle with the molar ratio of 0.1 as the optimal sample for further use hereafter.
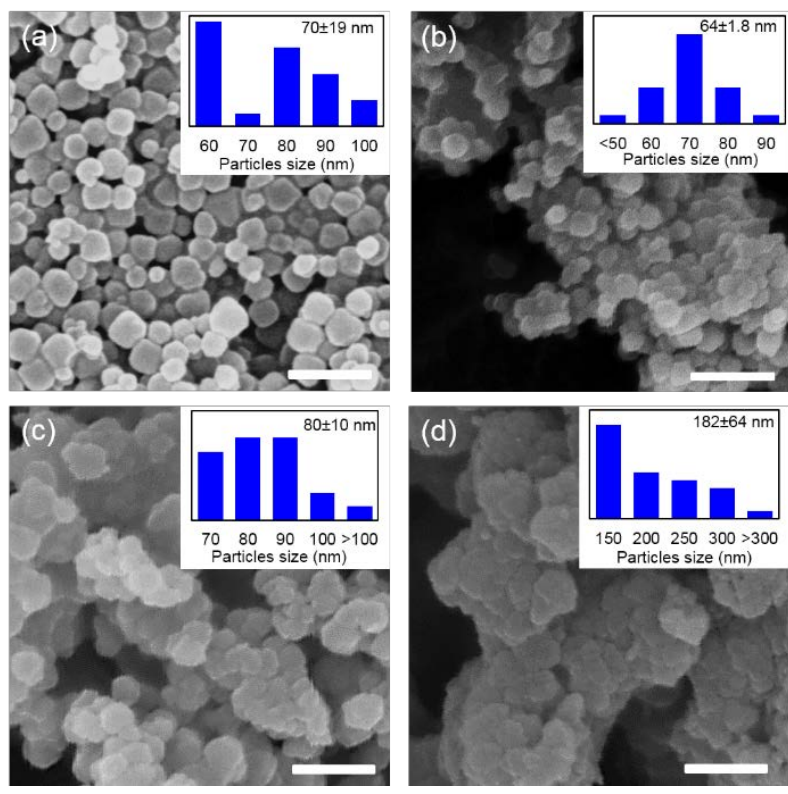

Figure 1. The SEM images of $\mathrm{AuCl}_{4}$ @ @ZIF-8 with different molar ratio of Au to Zn. (a) 0:1, (b) 0.01:1, (c) 0.1:1 and (d) 0.2:1. The scale bar is $250 \mathrm{~nm}$. The particle size distribution was calculated with TEM images $(n>100)$.

The as-synthesized $\mathrm{AuCl}_{4}^{-} @ \mathrm{ZIF}-8$ particles were collected and placed in a sodium borohydride solution to reduce $\mathrm{AuCl}_{4}{ }^{-}$ ions into Au metal, thus proving that $\mathrm{AuCl}_{4}^{-}$ions were indeed present inside the ZIF-8 nanoparticles. The obtained sample was referred to as Au@ZIF-8. As shown in Figure 2a, a typical TEM image of Au@ZIF-8 showed many black dots distributed inside each ZIF-8 nanoparticle. This indicated that gold nanoparticles could be successfully generated through our de novo method. The reduced Au nanoparticles exhibited a uniform particle size around $7.5 \pm 0.8 \mathrm{~nm}$ in diameter. Since this size is larger than the pore size of ZIF-8 $(1.1 \mathrm{~nm}),{ }^{[6]}$ it is not possible that the Au nanoparticles were synthesized outside and diffused into ZIF-8. Generally, the particle size of metal nanoparticles formed in confined space is smaller than those that weren't due to the rigid structure of the porous materials. ${ }^{[3]}$ However, the particle size of the gold nanoparticles formed in ZIF-8 is larger than the pore size of ZIF-8, which indicates that the cavity of ZIF-8 would be destroyed during the reduction of gold ion. Morabito et al. ${ }^{[38]}$ reported the same phenomenon and pointed out that ZIF-8 exhibits a flexible framework due to the dissociation and association of linkers in aqueous solution.
Meilikhov et al. ${ }^{[32]}$ also mentioned in their previous work that the metal nanoparticles synthesized inside MOF structure could
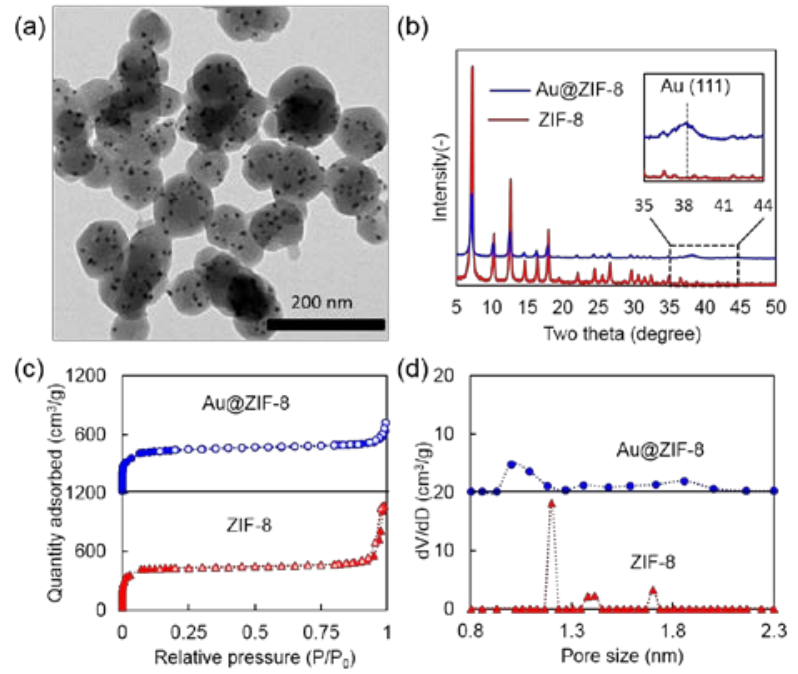

have particle size larger than the pore size of the MOF due to the partial destruction of the MOF framework.

Figure 2. (a) A TEM image of Au@ZIF-8. (b) XRD Patterns of Au@ZIF-8 and ZIF-8. (c) $N_{2}$ adsorption/desorption isotherms of Au@ZIF-8 and ZIF-8. (d) The corresponding pore size distribution of Au@ZIF-8 and ZIF-8.

The distortion of ZIF-8 crystalline framework after the formation of Au nanoparticles can also be evidenced by XRD. The XRD patterns of the final sample (i.e., Au@ZIF-8) and its parent sample (i.e., ZIF-8) are shown in Figure 2b. Each peak of Au@ZIF-8 shares the same position as that of pure ZIF-8, indicating that Au@ZIF-8 had the same crystalline phase as the pure ZIF-8. However, the broader and weaker XRD peaks of Au@ZIF-8 also indicate that the crystallinity of Au@ZIF-8 was not as good as that of pure ZIF-8. Meanwhile, an additional peak shown at $38.4^{\circ}$ represents the crystalline phase of (111) of gold, thus evidencing the existence of gold in Au@ZIF-8. The particle size of gold calculated from Scherrer's formula was around $5.7 \mathrm{~nm}$ which was smaller than that calculated from TEM image. The smaller size of Au NPs calculated from Scherrer's formula might be caused from the multi-crystals of gold inside ZIF-8.

The porous properties of the synthesized Au@ZIF-8 were analysed by nitrogen adsorption/desorption isotherms. As shown in Figure 2c and Table 1, the Au@ZIF-8 sample exhibited a typical type I isotherm with BET specific surface area of $1500 \mathrm{~m}^{2} / \mathrm{g}$, which is close to that of pure ZIF-8 (i.e., $1436 \mathrm{~m}^{2} / \mathrm{g}$ ). The corresponding pore size distribution of Au@ZIF-8 and ZIF-8 calculated by density functional theory are shown in Figure $\mathbf{2 d}$. The pore size (i.e., void) of ZIF-8 was found to be $1 \mathrm{~nm}$, which is close to the theoretical pore size of ZIF-8. ${ }^{[36]}$ However, the Au@ZIF-8 sample exhibited a smaller pore size than that of ZIF-8. Based on the calculation, we also found that the micropore volume of Au@ZIF-8 was greatly decreased, as compared to ZIF-8. This result also indicated that the synthesized Au nanoparticles occupied the voids inside the ZIF-8.

Although several papers have reported the synthesis of Au-loaded MOFs materials as efficient solid catalysts for various reactions, the molecular sizes of reactants are larger 
than the window size of MOFs. ${ }^{[39-41]}$ Such catalytic performance has been attributed to the fact that the synthesized $\mathrm{Au}$ nanoparticles were present not only inside the MOFs but also on the external surface of MOFs for additional catalysis
ZIF-8, indicating a successful and complete conversion of ZIF-8 to carbon. ${ }^{[21]}$ The porous properties of the Au@NC were measured and compared with those of ZIF-8 derived carbon. As shown in Figure $\mathbf{3 c}$ and Table 1, the specific surface areas

Table 1. Porous characteristics of samples.

\begin{tabular}{|ccccc|}
\hline Sample & $\begin{array}{c}\text { Specific surface } \\
\text { area }^{\mathrm{a}}\left(\mathrm{m}^{2} / \mathrm{g}\right)\end{array}$ & $\begin{array}{c}\text { Total pore volume } \\
\left(\mathrm{cm}^{3} / \mathrm{g}\right)\end{array}$ & $\begin{array}{c}\text { Micropore volume } \\
\left(\mathrm{cm}^{3} / \mathrm{g}\right)\end{array}$ & $\mathrm{V}_{\text {meso+macro }} / \mathrm{V}_{\text {micro }}$ \\
\hline ZIF-8 & 1436.2 & 1.662 & 0.584 & 1.85 \\
Au@ZIF-8 & 1500.4 & 1.107 & 0.099 & 10.18 \\
ZIF-8 der. C & 558.1 & 0.97 & 0.091 & 9.66 \\
Au@NC & 477.7 & 0.566 & 0.023 & 23.6 \\
\hline $\begin{array}{l}\text { a: The calculation of specific surface area was based on BET theory. } \\
\text { method. The calculation of total pore volume and micropore volume were based on } t \text {-plot }\end{array}$
\end{tabular}

contribution. Here, we

would like to confirm that the encapsulated Au nanoparticles mainly remained inside the ZIF-8 material, so we applied the synthesized Au@ZIF-8 as a heterogeneous catalyst for the reduction of 4-nitrophenol. If $\mathrm{Au}$ nanoparticles were indeed located inside ZIF-8, then no reaction should be observed because the molecular size of 4-nitrophenol (i.e., $0.64 \mathrm{~nm})^{[42]}$ is larger than the window size of ZIF-8 (i.e., $0.34 \mathrm{~nm}$ ). As shown in Figure S1, the Au@ZIF-8 exhibited a low conversion of 10\%, which was similar to that of ZIF-8 as the control sample. These results indicated that both ZIF-8 and Au@ZIF-8 had no ability to reduce 4-nitrophenol into 4-aminophenol. The $10 \%$ conversion from ZIF-8 and Au@ZIF-8 were resulted from the adsorption of 4-nitrophenol on the samples. To further confirm our hypothesis, we dissolved Au@ZIF-8 in $\mathrm{HCl}$ aqueous solution and collected the free $\mathrm{Au}$ nanoparticles for reaction. The free $\mathrm{Au}$ nanoparticles from Au@ZIF-8 showed 100\% conversion from 4-nitrophenol to 4-aminophenol, indicating that $\mathrm{Au}$ nanoparticles were present. Esken et al. ${ }^{[43]}$ have indicated that although ZIF-8 is a porous framework with high surface area, the small window size of ZIF-8 would inhibit the loading of catalytically active metal nanoparticles that usually have a larger size than that of the window. Therefore, conventional impregnation methods would cause the metal nanoparticles to form mostly on the external surface of ZIF-8. ${ }^{[29]}$ In contrast to these previous reports, we demonstrate that Au nanoparticles can be fully encapsulated inside ZIF-8 by our de novo synthesis.

To fully utilize the Au nanoparticles immobilized inside ZIF-8, it is necessary to enlarge the pore openings and to enhance the mechanical strength of the ZIF-8 support. We propose the direct conversion of ZIF-8 in the Au@ZIF-8 into nanoporous nitrogen-containing carbon (denoted as Au@NC) through pyrolysis (the detailed pyrolysis conditions are described in the Experimental section). As shown in Figure 3a, the TEM image of the Au@NC indicates that large pores could be generated through pyrolysis. The XRD pattern of Au@NC in Figure 3b suggest that the crystallinity of Au in Au@NC was better than that of Au in Au@ZIF-8, indicating that the pyrolysis process assisted the crystallization of the embedded $\mathrm{Au}$ nanoparticles. The particle size of Au in Au@NC was 5.7 nm from Scherrer's formula, and it was similar to that of Au@ZIF-8, indicating that the procedure of carbonization have no effect on gold nanoparticles. There were no characteristic peaks for of Au@NC and ZIF-8 derived carbon are 477 and $558 \mathrm{~m}^{2} / \mathrm{g}$, respectively. The corresponding pore size distribution of Au@NC and ZIF-8 derived carbon are shown in Figure 3d. In addition to several pores in the range of 0.5 to $2 \mathrm{~nm}$, there was a broad peak in the range of 2 to $6 \mathrm{~nm}$ for Au@NC. As mentioned before, the framework of ZIF-8 would be partially destroyed during the formation of Au nanoparticles. Therefore, this might be the reason that some mesopores were generated during the pyrolysis process. We also calculated the ratio of $\mathrm{V}_{\text {meso+macro }}$ to $\mathrm{V}_{\text {micro }}$ for all samples, and found that the Au@NC sample exhibited the highest $V_{\text {meso+macro }} / V_{\text {micro }}$ value among all samples. This indicated that Au@NC contained more macro- or mesopores, which are useful for the mass transport of reactants and products.

Figure 3. (a) A typical TEM image and (b) a XRD pattern of Au@NC. (c and (a)

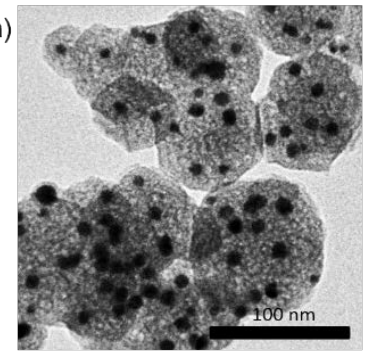

(c)

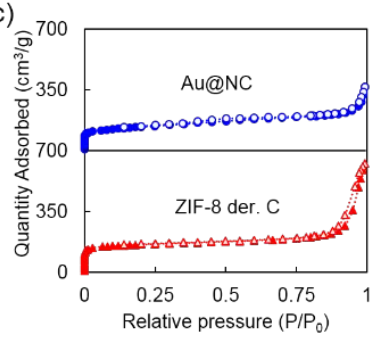

(b)

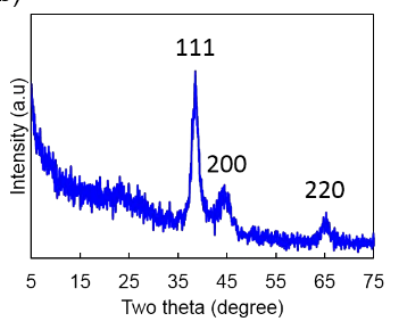

(d)

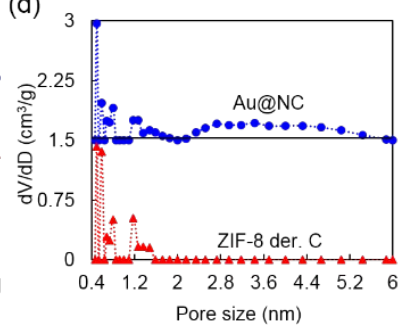

d) $\mathrm{N}_{2}$ adsorption/desorption and pore size distribution, respectively, of Au@NC and ZIF-8 derived carbon.

To demonstrate the catalytic ability of all samples, the conversion of 4-nitrophenol to 4-aminophenol was chosen as the model reduction reaction here. ${ }^{[44-47]}$ The UV/Vis spectra for the reduction of 4-nitrophenol catalyzed by Au@NC at different time periods are shown in Figure $\mathbf{4 a}$. The adsorption peaks at 
$400 \mathrm{~nm}$ and $305 \mathrm{~nm}$ resulted from 4-nitrophenol and 4-aminophenol, respectively. Therefore, it is clearly seen that as reaction time increased, the absorption intensity decreased at $400 \mathrm{~nm}$ and increased at $305 \mathrm{~nm}$, thus indicating a successful catalytic conversion of 4-nitrophenol to 4-aminophenol by Au@NC. In contrast, no catalytic performance was observed with Au@ZIF-8 (Figure S1). The efficient conversion using Au@NC indicated that the reactant (i.e., 4-nitrophenol with molecular size of $0.64 \mathrm{~nm}$ ) could penetrate into the nanoporous carbon framework and react with $\mathrm{Au}$ nanoparticles inside. The absorption of 4-nitrophenol appeared at $317 \mathrm{~nm}$. After the addition of a reducing agent (i.e., $\mathrm{NaBH}_{4}$ ), the 4-nitrophenol became 4-nitrophenolate ion with the corresponding absorption shifting to $400 \mathrm{~nm}$. The addition of Au@NC triggered the reduction of 4-nitrophenol, resulting in the absorption decreasing at $400 \mathrm{~nm}$ (the consumption of 4-nitrophenol) and increasing at $305 \mathrm{~nm}$ (the formation of 4-aminophenol).

(a)

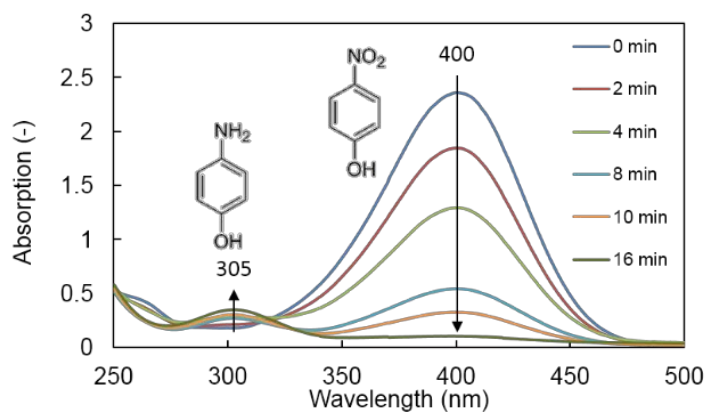

(b)

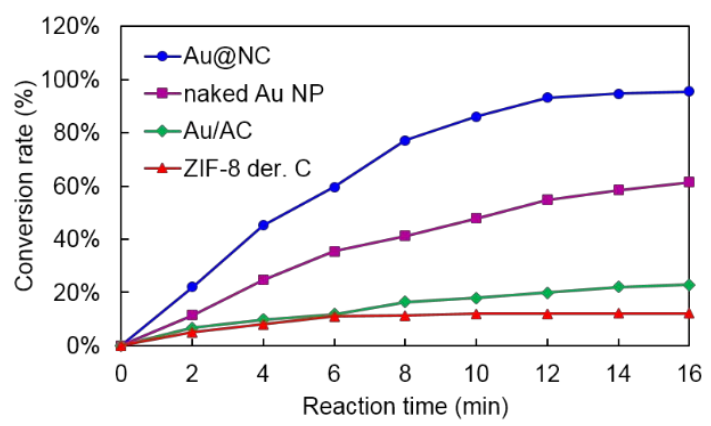

Figure 4. (a) UV-Vis spectroscopy for the reduction of 4-nitrophenol catalyzed by Au@NC at different time periods. (b) The reduction of 4-nitrophenol by ZIF-8 derived carbon, Au-loaded activated carbon (Au/AC), naked Au nanoparticles derived from Au@NC and Au@NC.

We further compared the catalytic ability of the synthesized Au@NC with other Au-based catalysts, including naked Au nanoparticles, Au-loaded activated carbon (Au/AC), and ZIF-8 derived carbon. For obtaining naked Au nanoparticles, Au@NC with the same amount used for catalysis was burned under air to remove the carbon. For preparing Au/AC, Au nanoparticles (3wt\%) were fixed on activated carbon. ZIF-8 derived carbon was used as the control sample. As shown in Figure $\mathbf{4 b}$, it is clearly seen that Au@NC exhibited the best performance among all catalysts. We calculated the rate constant and turnover frequency (TOF) from Figure 4b and Figure S3, with results summarized in Table 2. The rate constant and TOF of Au@NC were $0.2133 \mathrm{~min}^{-1}$ and $1185 \mathrm{~g}^{-1} \mathrm{~s}^{-1}$, respectively, which are higher than those of the other three samples.
The superior performance of Au@NC over naked Au nanoparticles is discussed as follows. First, we propose that the naked $\mathrm{Au}$ nanoparticles could not be suspended homogeneously in aqueous solution and would aggregate together because their surfaces were not covered with any capping agents such as polyvinylpyrrolidone or polyethylene glycol. The aggregation would then decrease the number of active sites on the surface, resulting in lower rate constant and TOF value. This result also emphasizes the necessity of a great support such as nanoporous carbon in this case for catalysis.

\begin{tabular}{|ccc|}
\hline $\begin{array}{c}\text { Table 2. } \\
\text { supports to reduce 4-nitrophenol into 4-aminophenol. } \\
\text { Sample }\end{array}$ & Rate constant $\left(\mathrm{min}^{-1}\right)$ & $\mathrm{TOF}^{\mathrm{a}}\left(\mathrm{g}^{-1} \mathrm{~s}^{-1}\right)$ \\
\hline Au@NC & 0.213 & 1185 \\
Naked Au NP & 0.061 & 339 \\
Au/AC & 0.016 & 89 \\
\hline
\end{tabular}

: The calculation of turnover frequency (TOF) was based on rate constant divided by the amount of gold on support.

Secondly, we discuss why our Au@NC had a better performance than the Au/AC catalyst. The surface of activated carbon is hydrophobic which is not appropriate for the impregnation of chloroauroic acid. We found that the adsorbed chloroauroic acid would form gold nanoparticles with larger and broader size distribution on activated carbon (Figure S3). The large and irregular particle size of $\mathrm{Au}$ nanoparticles would inhibit their efficiency (the same reason for naked $\mathrm{Au}$ nanoparticles). In addition, we also measured the zeta potentials of Au@NC (i.e., -8.2 mV) and Au/AC (i.e., -17.3 mV). The zeta potential of Au@NC was comparatively more positive than that of $\mathrm{Au} / \mathrm{AC}$, indicating that the negatively charged 4-nitrophenol would stay closer to Au@NC than to Au/AC. The 2-MIM of ZIF-8 provided the nitrogen atoms of Au@NC to contribute to a less hydrophobic and negatively charged surface, which are very useful for the adsorption of 4-nitrophenol. Many studies have learnt that nitrogen could provide positive charge and high spin density on the carbon surface, which changes the carbon surface from hydrophobic to hydrophilic. ${ }^{[48]}$ Kong et al. ${ }^{[48]}$ utilized $\mathrm{N}$-doped graphene to reduce 4-nitrophenol and found that negatively charged 4-nitrophenol could be easily adsorbed on positively charged $\mathrm{N}$-doped graphene via electrostatic interaction. All four types of nitrogen state (pyridinic, pyrrolic, amine, and graphitic) could enhance the adsorption of 4-nitrophenol to thus improve its conversion. Although 4-nitrophenol was adsorbed on metal but not carbon in our system, we suggest that the positively charged surface provided by nitrogen would increase the adsorption amount of 4-nitrophenol and thus enhance the catalytic reaction.

We analyse the hydrophobicity/hydrophilicity of Au@NC and Au/AC samples to prove our hypothesis. Hydro compatibility and element analysis of Au@NC and Au/AC are shown in Figure 5. $p$-Xylene and water are immiscible when mixed together, with $p$-xylene on the upper layer and water on the lower layer. When mixing Au/AC sample with the $p$-xylene/water solution, it was clearly seen in Figure $\mathbf{5 a}$ that the particles were dispersed in $p$-xylene (upper layer). On the contrary, the opposite was found when the Au@NC sample was added into the p-xylene/water solution (Figure $\mathbf{5 b}$ ). It has 
been known that most carbon-based materials are usually hydrophobic. However, our nitrogen-doped carbon material derived from ZIF-8 was hydrophilic. The compositions of activated carbon and ZIF-8 derived carbon were analysed with XPS. As shown in Figure 5, activated carbon mainly consists of carbon, oxygen, and silicon. In contrast, the ZIF-8 derived carbon is composed of carbon (62\%), nitrogen (31\%), and zinc $(6 \%)$. The nitrogen and zinc in the final carbon definitely resulted from 2-MIM, because the organic linker of ZIF-8 (i.e., 2-MIM) contains two nitrogen atoms and the basic unit of ZIF-8 is $\mathrm{Zn}_{6} \mathrm{~N}_{24} \mathrm{C}_{48} \mathrm{H}_{60}{ }^{[49]}$. Zhang et al. ${ }^{[21]}$ carbonized $\mathrm{ZIF-8}$ under different temperature and found that the atomic percentage of nitrogen would decrease gradually from $30 \%$ to $5 \%$ as the carbonization temperature increased. Maiyalagan and Viswanathan ${ }^{[50]}$ pointed out that the presence of nitrogen atoms in carbon framework could enhance the polarity, basicity, and hydrophilicity of the sample. On the other hand, near zero conversion of 4-nitrophenol by ZIF-8 and ZIF-8 derived carbon indicated that zinc would not be involved in the reaction. In our case, we believe that the enhanced hydrophilicity of Au@NC would help the adsorption of 4-nitrophenol and thus promote the catalytic reaction.

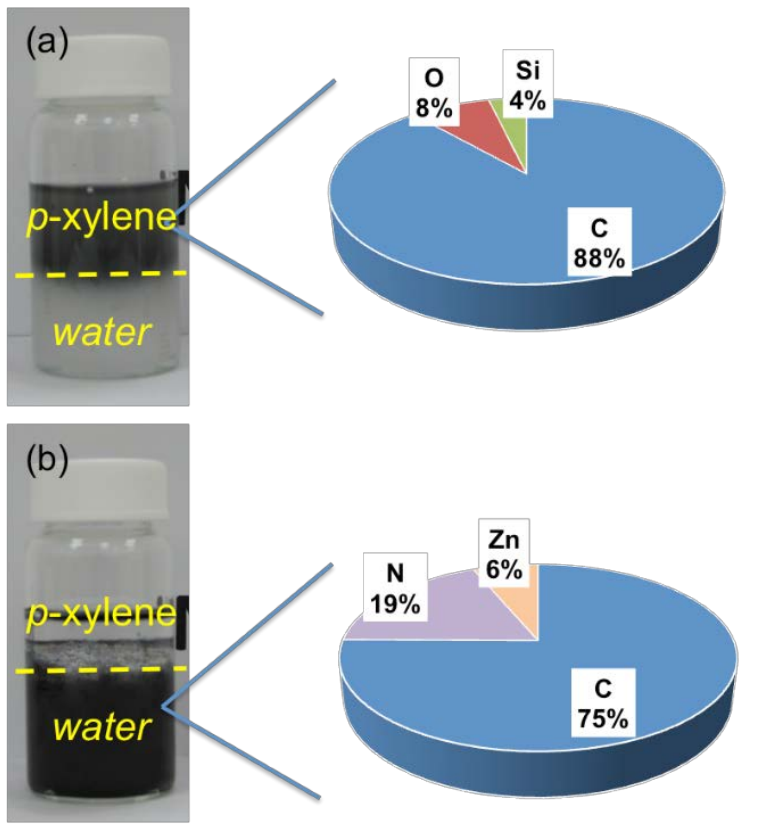

Figure 5. The hydro compatibility of ZIF-8 derived carbon (C) and activated carbon (AC). (a) Au/AC suspended and (b) Au@NC suspended mixture solution. The composition of carbon based structure was analyzed with XPS.

In summary, we demonstrate a de novo method to implant Au nanoparticles into ZIF-8 derived, nitrogen-containing nanoporous carbon (Au@NC) in aqueous systems. The synthesized Au@NC can serve as an efficient heterogeneous catalyst for converting 4-nitrophenol to 4-aminophenol. Our de novo method offers high percentage of $\mathrm{Au}$ nanoparticles embedded inside the ZIF-8 support. In addition, the carbonization of the nitrogen-containing ZIF-8 provides a mechanically stable carbon framework with abundant nitrogen atoms that generate a hydrophilic surface. Our study offers a novel tool to encapsulate and introduce other metal or metal oxide nanoparticles into nanoporous carbon materials, which would be useful in various catalytic applications.

\section{Experimental Section}

Chemicals 2-methylimidazole (2-MIM), zinc nitrate hexahydrate, chloroauric acid, and sodium borohydride were purchased from Sigma-Aldrich without further purification. 4-nitrophenol and 4-aminophenol were purchased from Acros. Deionized water was purified with a Milli-Q system (Millipore, Bedford, MA, USA).

Synthesis of zeolitic imidazolate framework-8 (ZIF-8) nanoparticles The procedure to synthesize ZIF-8 nanoparticles was modified from a previous paper. ${ }^{[51]}$ Briefly, 2-MIM (5.67 g) and zinc nitrate hexahydrate $(0.29 \mathrm{~g})$ were separately dissolved in deionized water $(20 \mathrm{~mL}$ and $2 \mathrm{~mL}$, respectively). After complete dissolution, the zinc nitrate solution was then poured into the 2-MIM solution, and the mixed solution was stirred for $10 \mathrm{~min}$. The white suspension was centrifuged at 20000 r.p.m. for $10 \mathrm{~min}$, and the precipitant was washed with water for 3 times. The white slurry after centrifugation was vacuum dried in lyophilizer and stored for further use.

De novo synthesis of Au nanoparticles embedded nanoporous carbon nanoparticles First, various amounts of chloroauric acid trihydrate was dissolved in deionized water (2 $\mathrm{mL}$ ) that contains zinc nitrate hexahydrate $(0.29 \mathrm{~g})$ with the final $\mathrm{Au} / \mathrm{Zn}$ ratio of $0: 1,0.01: 1,0.1: 1$, and $0.2: 1$. This solution was then poured into a 2-MIM (5.67 g in $20 \mathrm{~mL}$ deionized water) solution. The mixed solution was stirred for $10 \mathrm{~min}$, and the white suspension was centrifuged at 20000 r.p.m. for $10 \mathrm{~min}$ and washed with water for 3 times. The slurry was then suspended into $5 \mathrm{~mL}$ of deionized water (referred as $\mathrm{AuCl}_{4} @ \mathrm{ZIF}-8$ solution). For reduction of $\mathrm{Au}$ ions, a sodium borohydride (18 $\mathrm{mg}$ dissolved in $1 \mathrm{~mL}$ ionized water) solution was added into the $\mathrm{AuCl}_{4}{ }^{-} @ \mathrm{ZIF}-8$ solution, and the mixture was stirred for 3 hours. The dark violet suspension was then collected by centrifugation and washed with deionized water for 2 times. The dark violet slurry was then dried in lyophilizer and stored for further use.

To convert the ZIF-8 to nanoporous carbon nanoparticles, the synthesized Au@ZIF-8 samples were carbonized under $\mathrm{N}_{2}$ environment at $800{ }^{\circ} \mathrm{C}$ for 4 hours. The temperature gradient was set at $5{ }^{\circ} \mathrm{C} / \mathrm{min}$. The final product was referred to as nanoporous Au@NC.

Characterization The morphology and structure of the synthesized samples (i.e., ZIF-8, Au@ZIF-8, and nanoporous Au@NC) were observed with scanning electron microscopy (SEM, NovaTM NanoSEM 230), transmission electron microscopy (TEM, JEOL JEM-1200EX II), and X-ray diffractometer (XRD, Rigaku). The surface properties including specific surface area, pore size, and pore volume of the samples were measured with nitrogen adsorption/desorption analysis using ASAP 2020 micromeritics. The calculation of micropores was based on density functional theory (DFT). The actual concentration of gold in the samples was measured by ICP/MS (Agilent 7700e). The Au-containing samples (5 mg) were first calcined under air at $600{ }^{\circ} \mathrm{C}$ for $3 \mathrm{~h}$, and the residue was dissolved in aqua regia and then diluted in $2 \mathrm{wt} \%$ of nitric acid for analysis. The composition of carbon samples including the synthesized ZIF-8 derived carbon and commercial activated carbon was analyzed using X-ray photoelectron spectroscopy (XPS, Thermo Scientific, Theta Probe). The sample was grinded extensively and pressed into a translucent disc for analysis.

The hydrophobicity/hydrophilicity of the synthesized ZIF-8 derived carbon was measured as follows. $10 \mathrm{mg}$ of samples was mixed with $6 \mathrm{~mL}$ solution containing $3 \mathrm{~mL}$ of $p$-xylene and 3 $\mathrm{mL}$ of water. A commercial activated carbon was also used as a 
comparison. In addition, $2 \mathrm{mg}$ of samples was mixed with different kinds of $\mathrm{pH}$ indicator including Fast Garnet GBC $\left(\mathrm{pK}_{\mathrm{a}}\right.$ $2)$, methyl red ( $\mathrm{pK}_{\mathrm{a}}$ 4.8), nature red $\left(\mathrm{pK}_{\mathrm{a}} 6.8\right)$, and phenol red $\left(\mathrm{pK}_{\mathrm{a}} 7.65\right)$ to estimate the $\mathrm{pK}_{\mathrm{a}}$ of samples.

Reduction of 4-nitrophenol A 4-nitrophenol $(0.125 \mathrm{mM})$ aqueous solution was prepared for testing the reduction ability of all catalysts. Typically, the 4-nitrophenol solution $(15.4 \mathrm{~mL})$ was mixed with a fresh sodium borohydride aqueous solution $(1.32 \mathrm{M}, 2.35 \mathrm{~mL})$, denoted as Solution A. At the same time, the synthesized or commercial catalyst was suspended in aqueous solution with the concentration of $0.1 \mathrm{mg} / \mathrm{mL}$, denoted as Solution B. Then, $1 \mathrm{~mL}$ of Solution B was added into Solution A for catalytic reaction at various time periods. Every two minutes, $1 \mathrm{~mL}$ of mixture was taken and centrifuged at 14000 r.p.m, and the supernatant was measured with UV/Vis spectroscopy (Jasco V-670) at a wavelength of $400 \mathrm{~nm}$. The turn over frequency (TOF) calculation was based on the reaction rate as described by the equation below. The rate constant was the slope of $-\ln \left(C_{t} / C_{o}\right)$ vs. time $(t)$ and TOF could be estimated from the rate constant divided by the amount of gold used in reduction reaction.

$\frac{\mathrm{d} C_{t}}{\mathrm{dt}}=\mathrm{k} C_{t} C_{N a B H 4}$

\section{Acknowledgements}

We would like to thank the Ministry of Science and Technology (MOST), Taiwan and National Taiwan University (104R7706) for the funding support (103-2113-M-008-001; 104-2119-M-008-010). Y.Y. extends their sincere appreciation to the Deanship of Scientific Research at King Saud University for funding this Prolific Research Group (PRG-1436-19).

Keywords: De novo synthesis • gold nanoparticles •zeolitic imidazolate framework-8 $\bullet$ nanoporous carbon $\bullet$ 4-nitrophenol reductions

V. Polshettiwar, R. S. Varma, Green Chem. 2010, 12, 743-754 F. Lefebvre, J. M. Basset, J. Mol. Catal. A: Chem. 1999, 146, 3-12. C. Coperet, M. Chabanas, R. P. Saint-Arroman, J. M. Basset, Angew. Chem. Int. Ed. 2003, 42, 156-181.

V. Polshettiwar, C. Len, A. Fihri, Coord. Chem. Rev. 2009, 253, 2599-2626.

[5] C. DeCastro, E. Sauvage, M. H. Valkenberg, W. F. Holderich, J. Cat. 2000, 196, 86-94.

[6] D. Astruc, F. Lu, J. R. Aranzaes, Angew. Chem. Int. Ed. 2005, 44 7852-7872.

[7] M. V. Ganduglia-Pirovano, A. Hofmann, J. Sauer, Surf. Sci. Rep. 2007 $62,219-270$.

[8] S. Lim, K. Suh, Y. Kim, M. Yoon, H. Park, D. N. Dybtsev, K. Kim Chem. Commun. 2012, 48, 7447-7449.

[9] $\quad H$. Juntgen, Fuel 1986, 65, 1436-1446.

[10] O. M. Yaghi, M. O'Keeffe, N. W. Ockwig, H. K. Chae, M. Eddaoudi, J. Kim, Nature 2003, 423, 705-714.

[11] H. Furukawa, K. E. Cordova, M. O'Keeffe, O. M. Yaghi, Science 2013 $341,974$.

[12] A. Dhakshinamoorthy, A. M. Asiri, H. Garcia, Chem. Commun. 2014 50, 12800-12814

[13] J. Liu, D. M. Strachan, P. K. Thallapally, Chem. Commun. 2014, 50 466-468.

[14] D. W. Lim, J. W. Yoon, K. Y. Ryu, M. P. Suh, Angew. Chem. Int. Ed. 2012, 51, 9814-9817.

[15] L. Radhakrishnan, J. Reboul, S. Furukawa, P. Srinivasu, S. Kitagawa Y. Yamauchi, Chem. Mater. 2011, 23, 1225-1231.

[16] H. L. Jiang, B. Liu, Y. Q. Lan, K. Kuratani, T. Akita, H. Shioyama, F. Zong, Q. Xu, J. Am. Chem. Soc. 2011, 133, 11854-11857.

[17] D. Yuan, J. Chen, S. Tan, N. Xia, Y. Liu, Electrochem. Commun. 2009 11, 1191-1194.

[18] B. Liu, H. Shioyama, H. Jiang, X. Zhang, Q. Xu, Carbon 2010, 48 $456-463$.
[19] N. L. Torad, M. Hu, Y. Kamachi, K. Takai, M. Imura, M. Naito, Y. Yamauchi, Chem. Commun. 2013, 49, 2521-2523.

[20] L. He, L. Li, T. Wang, H. Gao, G. Li, X. Wu, Z. Su, C. Wang, Dalton Trans. 2014, 43, 16981-16985.

[21] L. Zhang, Z. Su, F. Jiang, L. Yang, J. Qian, Y. Zhou, W. Li, M. Hong, Nanoscale 2014, 6, 6590-6602.

[22] D. Esken, X. Zhang, O. I. Lebedev, F. Schroeder, R. A. Fischer, J. Mater. Chem. 2009, 19, 1314-1319.

[23] S. Hermes, M. K. Schroter, R. Schmid, L. Khodeir, M. Muhler, A. Tissler, R. W. Fischer, R. A. Fischer, Angew. Chem. Int. Ed. 2005, 44 6237-6241.

[24] R. J. T. Houk, B. W. Jacobs, F. El Gabaly, N. N. Chang, A. A. Talin, D. D. Graham, S. D. House, I. M. Robertson, M. D. Allendorf, Nano Lett 2009, 9, 3413-3418.

[25] M. D. Allendorf, R. J. T. Houk, L. Andruszkiewicz, A A Talin, J. Pikarsky, A. Choudhury, K. A. Gall, P. J. Hesketh, J. Am. Chem. Soc. 2008, 130, 14404-14405.

[26] G. Lu, S. Li, Z. Guo, O. K. Farha, B. G. Hauser, X. Qi, Y. Wang, X Wang, S. Han, X. Liu, J. S. DuChene, H. Zhang, Q. Zhang, X. Chen, J. Ma, S. C. J. Loo, W. D. Wei, Y. Yang, J. T. Hupp, F. Huo, Nat. Chem. 2012, 4, 310-316.

[27] P. Hu, J. V. Morabito, C. K. Tsung, ACS Catal. 2014, 4, 4409-4419.

[28] P. Hu, J. Zhuang, L. Y. Chou, H. K. Lee, X. Y. Ling, Y. C. Chuang, C. K. Tsung, J. Am. Chem. Soc. 2014, 136, 10561-10564.

[29] C. Roesler, R. A. Fischer, CrystEngComm 2015, 17, 199-217.

[30] Y. K. Hwang, D. Y. Hong, J. S. Chang, S. H. Jhung, Y. K. Seo, J. Kim, A. Vimont, M. Daturi, C. Serre, G. Ferey, Angew. Chem. Int. Ed. 2008, 47, 4144-4148.

[31] M. S. El-Shall, V. Abdelsayed, A. E. R. S. Khder, H. M. A. Hassan, H. M. El-Kaderi, T. E. Reich, J. Mater. Chem. 2009, 19, 7625-7631.

[32] M. Meilikhov, K. Yusenko, D. Esken, S. Turner, G. Van Tendeloo, R. A. Fischer, Eur. J. Inorg. Chem. 2010, 3701-3714.

[33] T. Tsuruoka, H. Kawasaki, H. Nawafune, K. Akamatsu, ACS Appl. Mater. Interfaces 2011, 3, 3788-3791.

[34] P. Wang, J. Zhao, X. Li, Y. Yang, Q. Yang, C. Li, Chem. Commun 2013, 49, 3330-3332.

[35] H. Guo, Y. Zhu, S. Wang, S. Su, L. Zhou, H. Zhang, Chem. Mater 2012, 24, 444-450.

[36] K. S. Park, Z. Ni, A. P. Cote, J. Y. Choi, R. Huang, F. J. Uribe-Romo, H. K. Chae, M. O'Keeffe, O. M. Yaghi, Proc. Natl. Acad. Sci. U.S.A. 2006 103, 10186-10191.

[37] L. Chen, H. Chen, Y. Li, Chem. Commun 2014, 50, 14752-14755.

[38] J. V. Morabito, L. Y. Chou, Z. Li, C. M. Manna, C. A. Petroff, R. J. Kyada, J. M. Palomba, J. A. Byers, C. K. Tsung, J. Am. Chem. Soc 2014, 136, 12540-12543.

[39] H. R. Moon, J. H. Kim, M. P. Suh, Angew. Chem. Int. Ed. 2005, 44 , 1261-1265.

[40] H. R. Moon, M. P. Suh, Eur. J. Inorg. Chem. 2010, 3795-3803.

[41] T. T. Dang, Y. Zhu, J. S. Y. Ngiam, S. C. Ghosh, A. Chen, A. M. Seayad, ACS Catal. 2013, 3, 1406-1410.

[42] A. V. Maffei, P. M. Budd, N. B. McKeown, Langmuir 2006, 22, 4225-4229.

[43] D. Esken, S. Turner, O. I. Lebedev, G. Van Tendeloo, R. A. Fischer, Chem. Mater. 2010, 22, 6393-6401.

[44] J. Zeng, Q. Zhang, J. Chen, Y. Xia, Nano Lett. 2010, 10, 30-35.

[45] J. Ge, T. Huynh, Y. Hu, Y. Yin, Nano Lett. 2008, 8, 931-934.

[46] A. K. Patra, A. Dutta, A. Bhaumik, Catal. Commun. 2010, 11, 651-655.

[47] N. Salam, B. Banerjee, A. S. Roy, P. Mondal, S. Roy, A. Bhaumik, S M. Islam, Appl. Catal., A 2014, 477, 184-194.

[48] X. K. Kong, Z. Y. Sun, M. Chen, C. I. Chen, Q. W. Chen, Energy Environ. Sci. 2013, 6, 3260-3266.

[49] H. Wu, W. Zhou, T. Yildirim, J. Am. Chem. Soc. 2007, 129, 5314-5315.

[50] T. Maiyalagan, B. Viswanathan, Mater. Chem. Phys. 2005, 93, 291-295.

[51] Y. Pan, Y. Liu, G. Zeng, L. Zhao, Z. Lai, Chem. Commun. 2011, 47, 2071-2073.

Received: ((will be filled in by the editorial staff))

Published online: ((will be filled in by the editorial staff)) 


\section{COMMUNICATION}

Nitrogen doped nanopourous carbon: Au embedded, nitrogen doped nanoporous carbon nanoparticles was successfully synthesized from zeolitic imidazolate framework-8 and utilized as reductive catalyst with enhanced reduction ability.

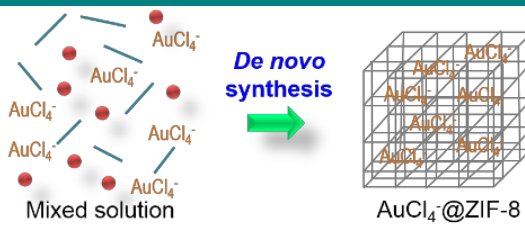

Reduction

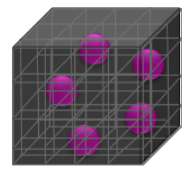

Au@NC
Carbonization

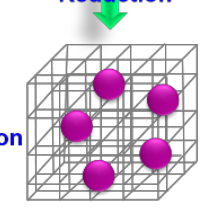

Au@ZIF-8
Yu-Te Liao, Jeffrey E. Chen, Yohei Isida, Tetsu Yonezawa, Wei-Chen Chang, Saad M. Alshehri, Yusuke Yamauchi, and Kevin C.-W. Wu*

Page No. - Page No.

De novo Synthesis of $\mathrm{Au}$ Nanoparticles-Embedded, Nitrogen-Doped Nanoporous Carbon Nanoparticles (Au@NC) with Enhanced Reduction Ability 